\title{
Observation of ionic Coulomb blockade
} in nanopores

\author{
Jiandong Feng ${ }^{1 \star}$, Ke Liu${ }^{1}$, Michael Graf', Dumitru Dumcenco ${ }^{2}$, Andras Kis ${ }^{2}$, Massimiliano Di Ventra ${ }^{3}$ \\ and Aleksandra Radenovic ${ }^{1 \star}$
}

Emergent behaviour from electron-transport properties is routinely observed in systems with dimensions approaching the nanoscale'. However, analogous mesoscopic behaviour resulting from ionic transport has so far not been observed, most probably because of bottlenecks in the controlled fabrication of subnanometre nanopores for use in nanofluidics. Here, we report measurements of ionic transport through a single subnanometre pore junction, and the observation of ionic Coulomb blockade: the ionic counterpart of the electronic Coulomb blockade observed for quantum dots. Our findings demonstrate that nanoscopic, atomically thin pores allow for the exploration of phenomena in ionic transport, and suggest that nanopores may also further our understanding of transport through biological ion channels.

Electrical transport through single molecules has attracted considerable attention ${ }^{2}$ owing to the possibility of using individual molecules as electronic components ${ }^{3}$. The first generation of singlemolecule electrical measurements was realized by probing transport in single quantum dots, carbon nanotubes, $\mathrm{C}_{60}$ molecules and DNA molecules using microfabricated structures, mechanically formed molecular junctions and scanning probe microscopies ${ }^{4}$. Such ultra-small platforms offer an ideal toolbox for probing electrons one at a time through the individual molecules, enabling direct observation of single-electron phenomena, such as Coulomb blockade, or the Kondo effect ${ }^{5}$ that have substantially contributed to our understanding of fundamental quantum physics and might lead to the realization of diverse quantum devices.

Analogous mesoscopic phenomena have also been predicted for ionic transport through narrow channels when they approach the dimensions of the hydration layers formed around each $\operatorname{ion}^{6,7}$. At very low densities one should then observe single-particle step-like conductance owing to the breaking of hydration layers as a function of channel radius ${ }^{6,7}$, whereas at larger densities ion-ion interactions should lead to the equivalent of Coulomb blockade when the channel has the appropriate capacitance ${ }^{6,8}$. However, owing to the requirement of controlled sub-nm channels, these phenomena have not been observed so far for ionic transport in nanofluidic systems ${ }^{9}$. Instead, ionic transport through nanochannels/nanopores typically exhibits ohmic behaviour. Voltage-activated nonlinear ion transport was also observed in various systems, such as hydrophobic wetting and ion dehydration ${ }^{10,11}$.

Here, we report for the first time the experimental observation of single-ion transport through the ionic junction of a sub-nm $\mathrm{MoS}_{2}$ pore. We observe that the current-voltage characteristics of ionic transport show nonlinear behaviour that exhibits a voltage gap at low applied bias. We suggest a single-ion transport mechanism based on single-ion charging, where the combination of Coulomb blockade and dehydration determines the ion transport through the sub-nm pore. Our observation is in a good agreement with predictions obtained for both solid-state pores $^{6}$ and a biological ion channel that has a similar geometry to the sub-nm solid-state nanopore reported here ${ }^{8}$, and it is reminiscent of the same phenomenon observed in electronic transport through quantum dots.

The experiments are conducted in a typical configuration where the two chambers with ionic solutions are separated by a single nanopore in a single-layer molybdenum disulfide $\left(\mathrm{MoS}_{2}\right)$ membrane $^{12}$ and biased by a pair of $\mathrm{Ag} / \mathrm{AgCl}$ electrodes, as described in Fig. 1a. The sub-nm nanopores used in this study are produced in a single-layer $\mathrm{MoS}_{2}$ membrane either by our recently reported atomic-scale nanopore fabrication technique using controlled electrochemical reaction or by electron irradiation within a Cs-corrected transmission electron microscope, as described elsewhere ${ }^{12,13}$. These techniques result in the opening of pores with diameters down to $0.3 \mathrm{~nm}$. From the plethora of twodimensional materials we have chosen 0.65 -nm-thick single-layer $\mathrm{MoS}_{2}$ (ref. 12), owing to the fact that it allows better control during nanopore fabrication ${ }^{12,13}$ and nanopores that exhibit better water transport when compared to graphene ${ }^{14}$. Fig. $1 \mathrm{~b}$ shows an image of pristine single-layer $\mathrm{MoS}_{2}$, a sulfur vacancy that acts as an initial site for nanopore formation during electrochemical reaction (Fig. 1c) and a 0.6-nm-diameter nanopore (Fig. 1d). For measurements in this work, we have mostly used pores fabricated using controlled electrochemical reaction ${ }^{13}$. The electrical characteristics of the pore are then determined by acquiring their current-voltage $(I-V)$ response in aqueous solutions of salts.

All $I-V$ measurements are performed at room temperature. Fig. 2a shows typical $I-V$ characteristics of a $0.6-\mathrm{nm}$ pore in potassium chloride $(\mathrm{KCl})$ aqueous solutions with a range of ionic strengths $(10 \mathrm{mM}-4 \mathrm{M})$. The curves exhibit a striking nonlinear behaviour, with an apparent gap of $\sim 400 \mathrm{mV}$. At small voltages, the current is suppressed. When the bias voltage exceeds the threshold value, the current starts to increase more strongly. Corresponding differential conductance $(\mathrm{d} I / \mathrm{d} V)$ plots are shown in Fig. 2b. Zero conductance is found in the gap region, as well as a conductance that increases with applied bias. This phenomenon differs largely from conventional ion transport through nanopores with larger sizes ${ }^{15}$, which can be described using classical Poisson-Nernst-Planck equations ${ }^{16}$. Representative $I-V$ curves from larger $\mathrm{MoS}_{2}$ pores $(d>1 \mathrm{~nm})$ are shown in Supplementary Fig. 1, exhibiting linear, ohmic responses. The response of another 0.6-nm $\mathrm{MoS}_{2}$ nanopore to different ion types is shown in Fig. 3. All tested metal ion types $\left(\mathrm{K}^{+}, \mathrm{Na}^{+}, \mathrm{Li}^{+}, \mathrm{Ca}^{2+}, \mathrm{Mg}^{2+}\right)$ exhibit nonlinearity in their

\footnotetext{
${ }^{1}$ Laboratory of Nanoscale Biology, Institute of Bioengineering, School of Engineering, EPFL, 1015 Lausanne, Switzerland. ${ }^{2}$ Laboratory of Nanoscale

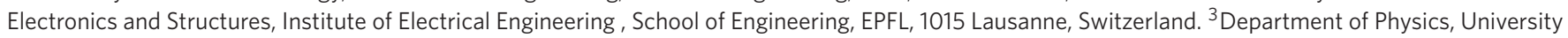
of California, San Diego, La Jolla, California 92093, USA. ^e-mail: jiandong.feng@epfl.ch; aleksandra.radenovic@epfl.ch
} 

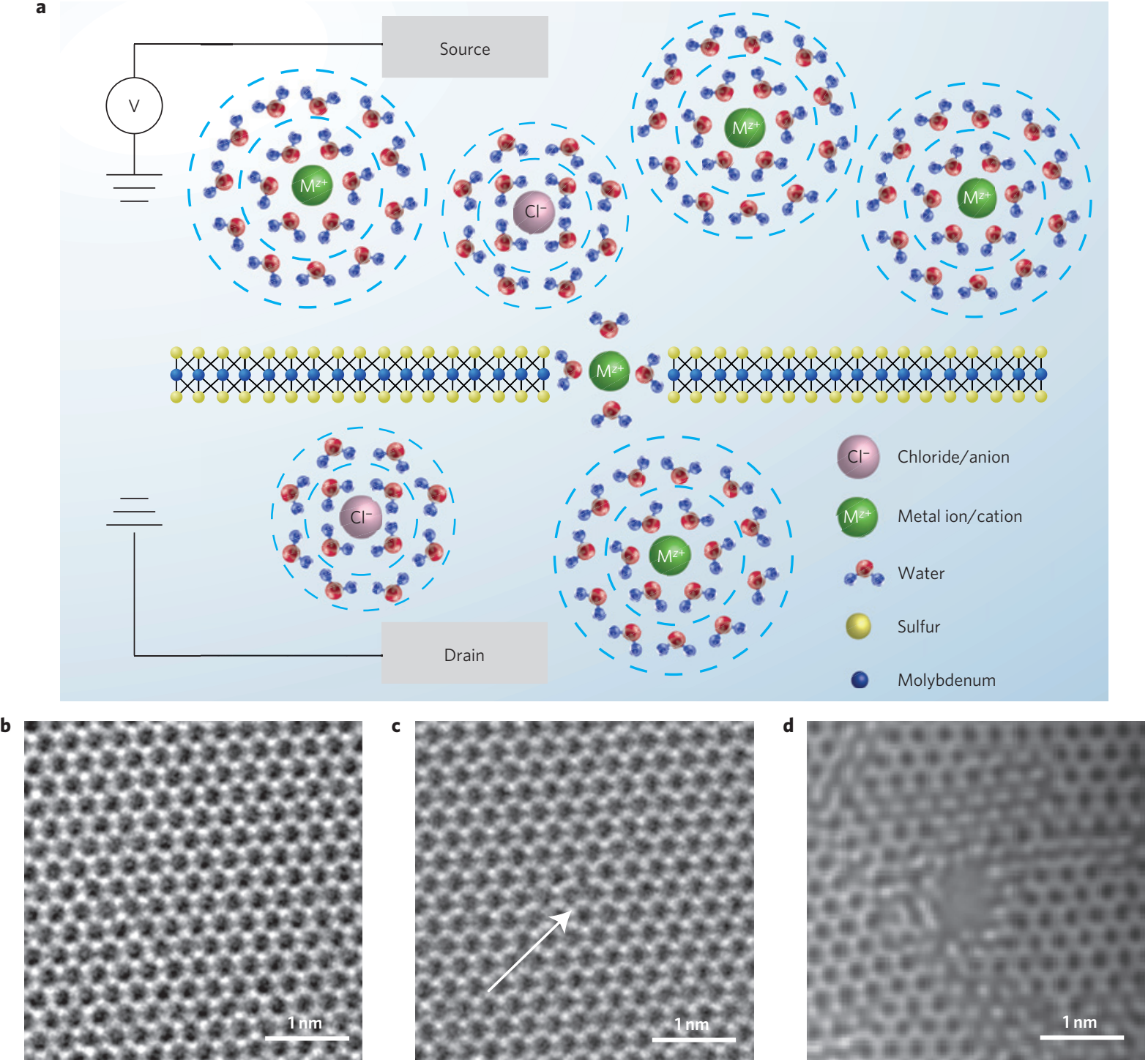

Figure 1 | lon transport through the sub-nm nanopore junction. a, Schematics of single-ion transport through a sub-nm MoS 2 nanopore.

b, Aberration-corrected transmission electron microscopy (TEM) image of a single-layer $\mathrm{MoS}_{2}$ membrane without a pore. c, Aberration-corrected TEM image of a single-atom vacancy (marked by arrow) in a single-layer $\mathrm{MoS}_{2}$ membrane. $\mathbf{d}$, Aberration-corrected TEM image of a 0.6-nm-diameter $\mathrm{MoS}_{2}$ nanopore.

$I-V$ characteristics (Fig. 3a), with different gap sizes for different ions and a strong dependence on the valence (referring to the differential conductance shown in Fig. 3b). Compared to other ions, the $I-V$ characteristic of the divalent ions $\mathrm{Mg}^{2+}$ and $\mathrm{Ca}^{2+}$ shows a more suppressed current for positive voltages than the monovalent ions $\mathrm{K}^{+}, \mathrm{Na}^{+}$and $\mathrm{Li}^{+}$. The hydrophilic nature of $\mathrm{MoS}_{2}$ pores owing to the Mo-rich pore surface and the pore wetting procedure we use, which involves soaking nanopores in an ethanol/water mixture, eliminates the possibility that nonlinearity originates from hydrophobic effects ${ }^{10,17}$.

To understand the nature of the ionic transport measurements, we first used an analogy to electric transport through a quantum dot/single-molecule system ${ }^{4}$, shown in Supplementary Fig. 2. Here, the small capacitance of a system weakly coupled to electrodes through tunnel junctions gives rise to Coulomb blockade ${ }^{18,19}$. In the schematics in Fig. 2c,d, and the energy diagrams in Fig. 2e, we attempt to define the 'electronic structure' of the single-ion channel device and employ the Coulomb blockade theory for ionic transport. In addition to a resistance, a nanopore junction also has a capacitance. A charging energy then needs to be overcome when the capacitance of the system is small enough such that many-body ion-ion interaction effects dominate. Therefore, the suppressed conductance in the subthreshold regime is a consequence of the finite energy required to add a charge carrier (ion) to the pore. Referring to the Coulomb blockade mode ${ }^{20}$ this addition energy $\left(\Delta_{N+1}\right)$ is given by

$$
\Delta_{N+1}=\mu_{N+1}-\mu_{N}=U+\Delta E
$$

where $\mu_{N}$ is the electrochemical potential of an $N$-ion system, $U$ is the Coulomb interaction energy between two ions in the pore and $\Delta E$ is the electronic excitation energy.

The ionic Coulomb interaction can be estimated using the electrostatic self-energy ${ }^{21}$ by considering a one-dimensional Coulomb gas model of ions ${ }^{22}$. Following the ionic Coulomb blockade model developed by Kaufman et al. ${ }^{8}$, the Coulomb gap can be obtained as a function of pore geometry and ion valence ${ }^{8,22}$,

$$
U=\frac{z^{2} e^{2}}{2 C}=\frac{1}{4 \pi \varepsilon_{0}} \frac{z^{2} e^{2} L}{2 \varepsilon_{\mathrm{W}} r^{2}}
$$



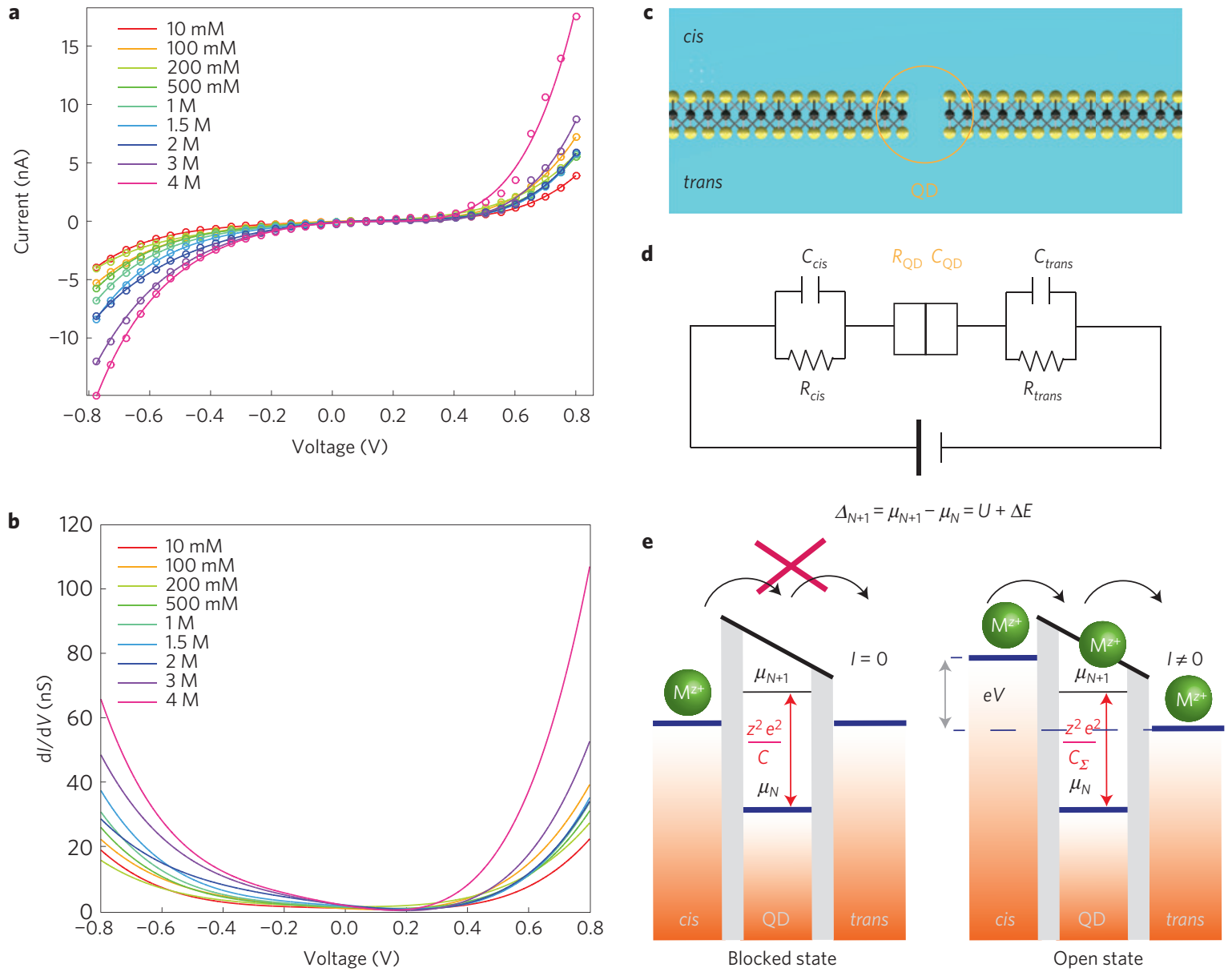

Figure 2 | Current-voltage characteristics (I-V) of a 0.6-nm $\mathrm{MoS}_{2}$ nanopore in a potassium chloride aqueous solution $(\mathrm{KCl})$. a, $I-V \mathrm{curves}$ acquired under different ion concentrations. $\mathbf{b}$, Differential conductance (dl/dV) of a $0.6-\mathrm{nm} \mathrm{MoS}$ in $\mathrm{KCl}$ solutions of different molarities. $\mathbf{c}$, Illustration of a single-ion junction in the form of small-diameter nanopores in the two-dimensional material $\mathrm{MoS}_{2}$. An analogy to quantum dot, QD, is used to describe the nanopore ion junction. $\mathbf{d}$, Corresponding equivalent circuit for the system shown in c. $C$, capacitance; $R$, resistance. e, Equivalent energy-level diagram for ionic Coulomb blockade due to single-ion charging in nanopores. With the sub-nm pore configuration presented, this charging energy is much larger than the thermal energy $\left(k_{B} T\right)$, otherwise the ion will be able to pass by means of thermal excitation. lonic conduction is suppressed (blocked state, left) when the applied bias (eV) is below the charging energy. A build-up in voltage above the charging energy allows conduction (open state, right) with a strong dependence on ion valence number $z$.

where $e$ is the elementary charge; $\varepsilon_{0}$ is vacuum dielectric constant and $\varepsilon_{\mathrm{w}}$ is the water dielectric constant; $C$ is the geometry-dependent self-capacitance ${ }^{22}$ of the pore, given by $4 \varepsilon_{\mathrm{w}} \varepsilon_{0} \pi r^{2} / L ; z$ is the ion valence number; $r$ is the radius of the pore and $L$ is its length. This model highlights two important contributions that lead to ionic Coulomb blockade: a geometry-dependent self-capacitance similar to electronic Coulomb blockade and an ion valence which is unique in ionic transport. Given the verified thickness of $\mathrm{MoS}_{2}$ nanopores and a 0.6- $\mathrm{nm}$ pore diameter, a Coulomb energy of about $3 k_{\mathrm{B}} T$ for a $0.65-\mathrm{nm}$ theoretical single-layer thickness and $5 k_{\mathrm{B}} T$ for a $1.6-\mathrm{nm}$ hydration thickness ${ }^{12}$ can be estimated for monovalent ions. For divalent ions, these values rise considerably to $12 k_{\mathrm{B}} T$ and $20 k_{\mathrm{B}} T$. A detailed estimation of the Coulomb gap for various pore diameters is given in Supplementary Table 1. The pore capacitance can also be extracted from the currentmolarity relation ${ }^{6}$. A non-monotonic current-molarity relation is observed, as a direct result of the capacitance effect of the pore ${ }^{6}$. By fitting to the classical ionic Coulomb blockade model ${ }^{6}$ using our device geometry and experimental results presented in Fig. 2, we obtain a pore capacitance of about $0.2 \mathrm{aF}$ for the $0.6-\mathrm{nm}$ pore, which is very close to our estimate, as shown in Supplementary Fig. 3. This non-monotonic behaviour is also predicted where the self-energy barrier versus concentration also shows a characteristic sharp dip in the vicinity of a certain negative monovalent dopant concentration, explained as a transition between different states $^{23}$. The second effect that contributes directly to the observed energy gap is the dehydration of ions in nanopores smaller than $0.8 \mathrm{~nm}$. This contribution becomes significantly large, and a stepwise free energy barrier originating from successive breaking of the third, second and first hydration shells is introduced when reducing the pore size from $0.8 \mathrm{~nm}$ to zero $^{7}$. Therefore, the dehydration effect should also be incorporated in the energy gap. To this end, we again used an analogy to the combined gap in the single-molecule system and in the ionic case; the 'energy-level spacing' is treated using the hydration energy. The estimated contribution from the ionic Coulomb blockade effect and the dehydration effect is shown in Supplementary Table 1 and Supplementary Fig. 4, and the Coulomb energy is found to be the main contribution in the pore size range 0.6 to $1 \mathrm{~nm}$. In Supplementary Fig. 4, we hypothesize a transition of the role from dehydration $(<0.5 \mathrm{~nm})$ to ion Coulomb blockade (in the range in which we work, 0.6 to $1 \mathrm{~nm}$ ). The single-particle dehydration effect is thus easily incorporated in the standard model of Coulomb blockade. 
a

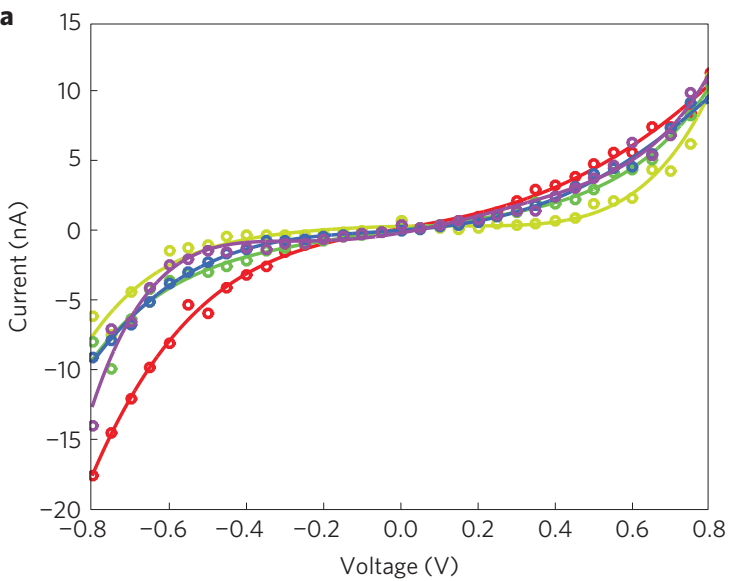

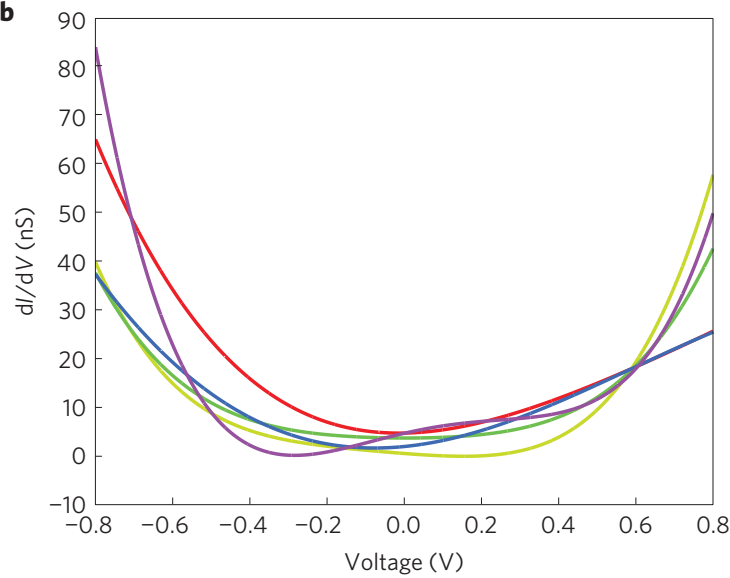

$-1 \mathrm{M} \mathrm{CaCl}_{2}=1 \mathrm{M} \mathrm{KCl}=1 \mathrm{M} \mathrm{LiCl}=1 \mathrm{M} \mathrm{MgCl}_{2} \quad-1 \mathrm{M} \mathrm{NaCl}^{2}$

Figure 3 | Valence-dependent ion transport. a, I-V curves of a $0.6-n m \mathrm{MoS}_{2}$ pore acquired in different salt solutions $\left(\mathrm{K}^{+}, \mathrm{Na}^{+}, \mathrm{Li}^{+}, \mathrm{Ca}^{2+}, \mathrm{Mg}^{2+}\right)$. b. Corresponding differential conductance $\mathrm{d} / \mathrm{d} \mathrm{d}$ plots. $\mathrm{Mg}^{2+}$ and $\mathrm{Ca}^{2+}$ have a more suppressed current for positive voltages.

This charging energy is much larger than the thermal energy at room temperature $\left(k_{\mathrm{B}} T, 298 \mathrm{~K}\right)$, allowing the direct observation of Coulomb blockade in ionic transport at room temperature. An applied bias $(\mathrm{eV})$ can adjust the chemical potential of the electrode, so as to add or remove a single charge carrier from the pore. When this energy rises above the Coulomb gap, the ion flux is able to pass through the pore. Measured values are in good agreement with the results from the calculations outlined above. A small deviation can be expected, due to the overestimation of the pore size and the additional capacitance between the pore and electrode. The valence dependence, where divalent ions show a slightly larger gap in the positive voltage direction than monovalent ions, is a direct consequence of the stronger Coulomb interaction between divalent ions, as shown in Fig. 3. This suppression is much lower than the straightforward estimation, and we believe that it is due to the combined result of the gap from both divalent cations $\left(\mathrm{Mg}^{2+} / \mathrm{Ca}^{2+}\right)$ and monovalent anions $\left(\mathrm{Cl}^{-}\right)$. The observed $I-V$ rectification might originate from the dipolar nature of $\mathrm{MgCl}_{2} / \mathrm{CaCl}_{2}$ due to the difference between the transport barrier for divalent cations and monovalent anions. In addition, a similar phenomenon where the self-energy barrier of $\mathrm{CaCl}_{2}$ exhibits the same barrier as $\mathrm{Na}^{+}$is also theoretically predicted, and explained by the fractionalization of divalent cations $\left(\mathrm{Mg}^{2+} / \mathrm{Ca}^{2+}\right)$ on two unit-charge mobile solitons ${ }^{23}$.

To observe Coulomb oscillations in most traditional electrical devices $^{20}$, one would need to incorporate a voltage gate. Nevertheless, integration of the voltage gate in the atomically thick pore requires passivation with a thick layer of dielectric to reduce current crosstalk $^{24}$. In turn, this requirement would compromise the atomic dimensions of our present nanofluidic device. Alternatively, in nanofluidic devices, gating can be realized by using the $\mathrm{pH}$ to modulate the surface charge of the system ${ }^{25}$. The surface chemistry of the $\mathrm{MoS}_{2}$ nanopore determines the negatively charged surface and an increase in $\mathrm{pH}$ will lead to the addition of negative charges. The ionic transport can then be modulated by the surface charge. Figure $4 \mathrm{a}$ shows the $\mathrm{pH}$ (2.5 to 11) dependence of conduction in our system. A non-monotonic dependence of pore conductance on $\mathrm{pH}$ is observed, and conduction peaks are found close to $\mathrm{pH} 3.5$ and $\mathrm{pH} 6$, which correspond to a certain integral number of excess charges in the pore, determined by the surface chemistry. However, the various possibilities of pore chemistry at the single-atom level ${ }^{14}$ can lead to variations in the conduction peaks from pore to pore, as shown in Supplementary Fig. 5. Kaufman et al. ${ }^{8}$ also modelled pore-charge-induced
Coulomb blockade oscillations by approximating the self-energy, $U_{n}=Q_{n}^{2} / 2 C$, of excess charge (where $Q_{n}$ represents the excess charge at the pore for the $n$ ions as a function of $Q_{f}, Q_{n}=z e n+Q_{f}$ ) and applying Fermi-Dirac occupancy statistics. The blockade region is found around a neutralized excess charge, and barrier-less conduction appears at the crossover point where $U_{n}=U_{n+1}$, which corresponds to the conductance oscillations we observed at certain $\mathrm{pH}$ values.

The Coulomb gap of the single-ion channel can be further engineered by adjusting the pore size. In the absence of the pore, the defect-free membrane is almost insulating, and the leakage current is very low (pA range). The smallest nanopore from a single vacancy with either two sulfur atoms or one molybdenum atom missing would produce a large gap, providing this structure is thermodynamically stable in liquid ${ }^{26}$. Single vacancies can also be generated using our controlled electrochemical pore fabrication method. However, it is difficult to measure the gap of a single vacancy $(d<3 \AA)$ in practice because a bias voltage higher than $0.8 \mathrm{~V}$ can result in the electrochemical dissolution of $\mathrm{MoS}_{2}$. We find only a handful of freestanding, defect-free $\mathrm{MoS}_{2}$ membranes suspended over small windows $(<50 \mathrm{~nm})$ that can operate up to $5 \mathrm{~V}$. Supplementary Fig. 6 gives an example of an $I-V$ measurement performed on a $0.3-\mathrm{nm}$ pore. A larger gap of $\sim 1.2 \mathrm{~V}$ is observed, and the voltage is swept in both directions. Cyclic $I-V$ measurements are taken to prevent the enlargement of the existing pore or the formation of a new pore in the presence of a high bias. We suspect the dehydration of a strong second hydration shell also plays an important role here. The measured gap size decreases with increasing pore size, and the charging energy can be obtained directly from the gap. As shown in Fig. 4c-e, a linear transition point is found near $1 \mathrm{~nm}$, indicating the disappearance of the transport barrier, with the $I-V$ characteristic becoming linear after this point. The strong correlation between the nonlinearity of the currentvoltage characteristics and the pore dimension further confirms that ionic Coulomb blockade is at the origin of the observed effect. With larger pores, the weak Coulomb interaction could in principle be measured at low temperatures, and even Coulomb stairs might be observed at low voltages. However, operation at low temperatures is limited by the liquid environment itself. Single-step transition from an insulating regime dominated by Coulomb blockade to a semiconducting regime has been observed previously in metallic quantum $\operatorname{dots}^{27,28}$

The prediction of ion transport through a symmetric biological ion channel has shown Coulomb blockade with a very similar 


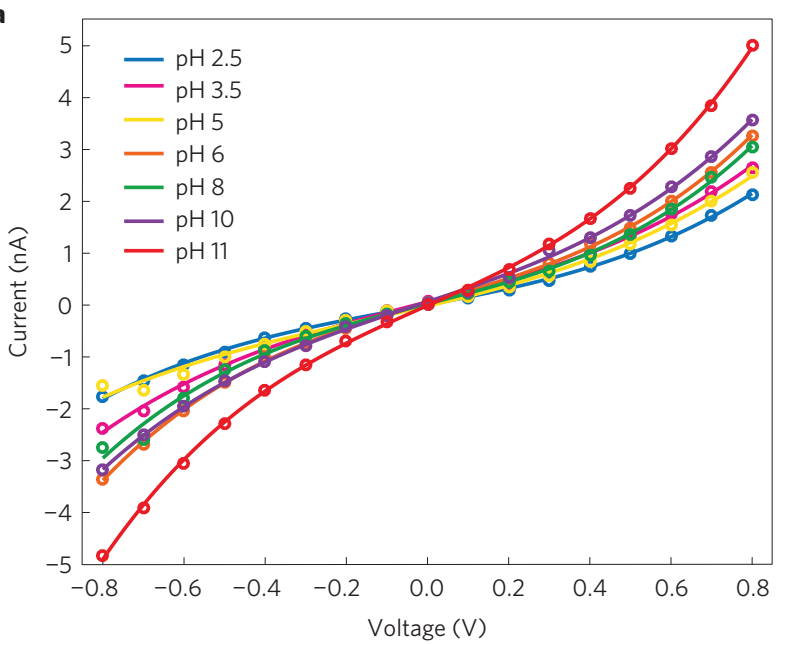

b

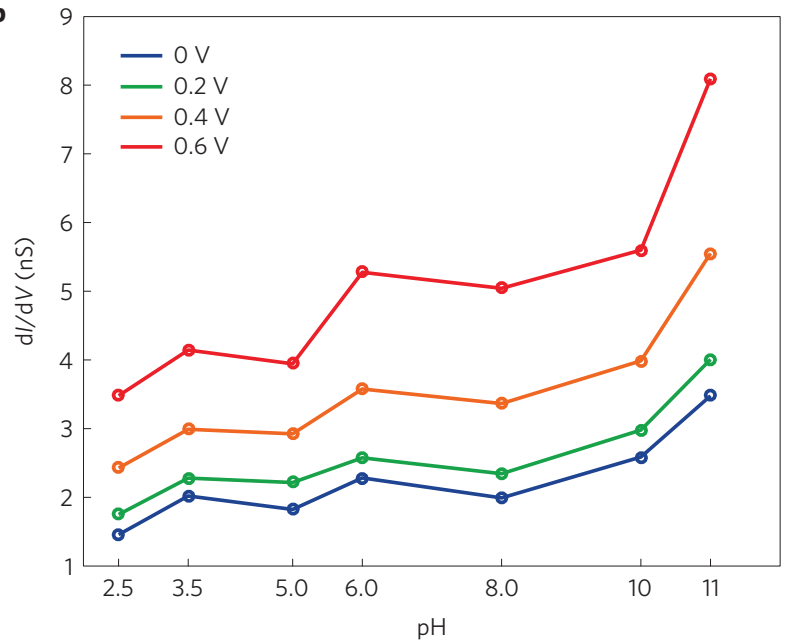

c

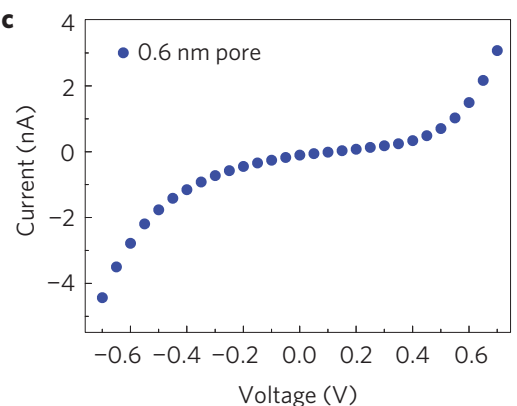

d

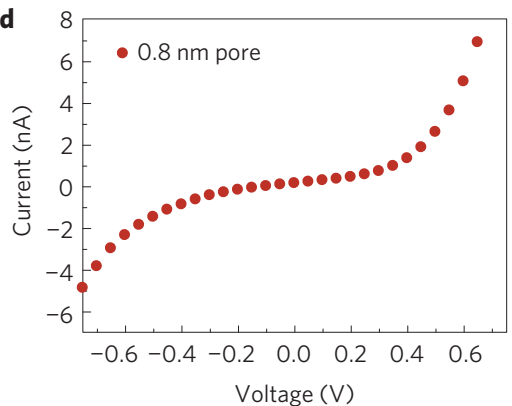

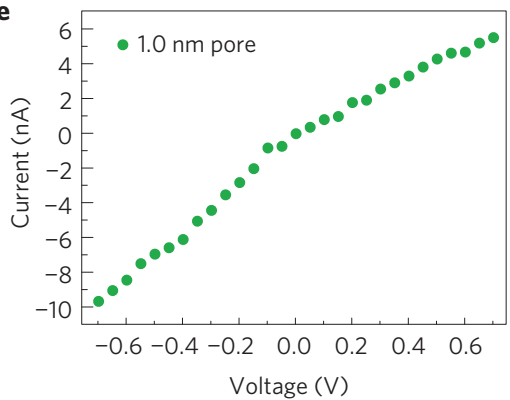

Figure 4 | pH-induced conductance oscillations. a, $\mathrm{pH}$-gated ionic transport through a $0.8-\mathrm{nm} \mathrm{MoS}$ nanopore. I- $\mathrm{V}$ curves are taken in various $\mathrm{pH}$ conditions of $1 \mathrm{M} \mathrm{KCl}(\mathrm{pH} 2.5$ to 11). $\mathbf{b}, \mathrm{dl} / \mathrm{d} V$ curves acquired in various $\mathrm{pH}$ conditions of $1 \mathrm{M} \mathrm{KCl}(\mathrm{pH} 2.5$ to 11). The differential conductance exhibits a non-monotonic behaviour with $\mathrm{pH}$ (surface charge). c-e, Variation of the linear-nonlinear transition with changing pore size, $0.6 \mathrm{~nm}(\mathbf{c}), 0.8 \mathrm{~nm}(\mathbf{d})$ and $1 \mathrm{~nm}(\mathbf{e})$. The gap size decreases with the pore size, and disappears when the pore size reaches $1 \mathrm{~nm}$.

geometry, pore size of $0.6 \mathrm{~nm}$ and thickness of $1.6 \mathrm{~nm}$ (ref. 8). The good agreement between the above prediction and experimental results further strengthens our claims. Finally, although Coulomb blockade $^{8}$ (a many-body effect) is concomitant with the singleparticle dehydration effect ${ }^{7}$, the following four observations support the contribution from Coulomb blockade: the low capacitance of the experimental configuration, the valence dependence, the peaks in current-molarity relation, and the conductance oscillations from $\mathrm{pH}$-induced surface charge modulation (Supplementary Information, Coulomb blockade discussion).

The observation of unique ionic transport in sub-nm pores is, to our knowledge, the first instance of a quantum-like mesoscopic effect dominating ionic transport. The measured nonlinear currentvoltage dependence for individual sub-nm pores is a consequence of single-ion charging behaviour, as described by the ionic Coulomb blockade model $^{8}$. Other possibilities that may also introduce a transport barrier, such as a dehydration effect, could also be further incorporated in the standard model. Our findings can be applied to nanopores with similar geometries. Our measurements provide direct experimental evidence that the effects observed here should also play a role in the functioning of biological ion channels, such as voltage-gated ion channels, as suggested in the theoretical model proposed by Kaufman and colleagues ${ }^{8}$. Future experiments investigating various gating effects, combined with better theoretical modelling, are needed to elucidate the results reported here. The blockade region also adds a new dimension to practical applications, such as water desalination ${ }^{29}$. We believe that atomically thin and small $(<1 \mathrm{~nm})$ pores offer a new platform from which to explore novel physics in the research areas of both nanoscale fluidics and biology.

\section{Methods}

Methods and any associated references are available in the online version of the paper.

Received 23 September 2015; accepted 24 February 2016; published online 28 March 2016

\section{References}

1. Datta, S. Electronic Transport in Mesoscopic Systems (Cambridge Univ. Press, 1997).

2. Perrin, M. L., Burzuri, E. \& van der Zant, H. S. J. Single-molecule transistors Chem. Soc. Rev. 44, 902-919 (2015).

3. Aviram, A. \& Ratner, M. A. Molecular rectifiers. Chem. Phys. Lett. 29, 277-283 (1974).

4. Nitzan, A. \& Ratner, M. A. Electron transport in molecular wire junctions. Science 300, 1384-1389 (2003).

5. Park, J. et al. Coulomb blockade and the Kondo effect in single-atom transistors. Nature 417, 722-725 (2002).

6. Krems, M. \& Di Ventra, M. Ionic coulomb blockade in nanopores. J. Phys. Condens. Matter 25, 065101 (2013).

7. Zwolak, M., Lagerqvist, J. \& Di Ventra, M. Quantized ionic conductance in nanopores. Phys. Rev. Lett. 103, 128102 (2009).

8. Kaufman, I. K., McClintock, P. \& Eisenberg, R. Coulomb blockade model of permeation and selectivity in biological ion channels. New J. Phys. 17, 083021 (2015)

9. Sparreboom, W., van den Berg, A. \& Eijkel, J. C. T. Principles and applications of nanofluidic transport. Nature Nanotech. 4, 713-720 (2009).

10. Powell, M. R., Cleary, L., Davenport, M., Shea, K. J. \& Siwy, Z. S. Electric-field-induced wetting and dewetting in single hydrophobic nanopores. Nature Nanotech. 6, 798-802 (2011).

11. Jain, T. et al. Heterogeneous sub-continuum ionic transport in statistically isolated graphene nanopores. Nature Nanotech. 10, 1053-1057 (2015). 
12. Liu, K., Feng, J. D., Kis, A. \& Radenovic, A. Atomically thin molybdenum disulfide nanopores with high sensitivity for DNA translocation. ACS Nano 8 , 2504-2511 (2014).

13. Feng, J. et al. Electrochemical reaction in single layer $\mathrm{MoS}_{2}$ : nanopores opened atom by atom. Nano Lett. 15, 3431-3438 (2015).

14. Farimani, A. B., Min, K. \& Aluru, N. R. DNA base detection using a single-layer $\mathrm{MoS}_{2}$. ACS Nano 8, 7914-7922 (2014).

15. Ho, C. et al. Electrolytic transport through a synthetic nanometer-diameter pore. Proc. Natl Acad. Sci. USA 102, 10445-10450 (2005).

16. Cervera, J., Schiedt, B. \& Ramirez, P. A Poisson/Nernst-Planck model for ionic transport through synthetic conical nanopores. Europhys. Lett. 71, 35-41 (2005).

17. Radenovic, A., Trepagnier, E., Csencsits, R., Downing, K. H. \& Liphardt, J. Fabrication of $10 \mathrm{~nm}$ diameter hydrocarbon nanopores. Appl. Phys. Lett. 93, 183101 (2008).

18. Beenakker, C. W. J. Theory of Coulomb-blockade oscillations in the conductance of a quantum dot. Phys. Rev. B 44, 1646-1656 (1991).

19. Fulton, T. A. \& Dolan, G. J. Observation of single-electron charging effects in small tunnel-junctions. Phys. Rev. Lett. 59, 109-112 (1987).

20. Kouwenhoven, L. P. et al. in Mesoscopic Electron Transport (eds Sohn, L. L., Kouwenhoven, L. P. \& Schön, G.) 105-214 (Kluwer, 1997).

21. Parsegian, A. Energy of an ion crossing a low dielectric membrane: solutions to four relevant electrostatic problems. Nature 221, 844-846 (1969).

22. Zhang, J., Kamenev, A. \& Shklovskii, B. I. Conductance of ion channels and nanopores with charged walls: a toy model. Phys. Rev. Lett. 95, 148101 (2005).

23. Zhang, J., Kamenev, A. \& Shklovskii, B. Ion exchange phase transitions in water-filled channels with charged walls. Phys. Rev. E 73, 051205 (2006).

24. Traversi, F. et al. Detecting the translocation of DNA through a nanopore using graphene nanoribbons. Nature Nanotech. 8, 939-945 (2013).

25. Stein, D., Kruithof, M. \& Dekker, C. Surface-charge-governed ion transport in nanofluidic channels. Phys. Rev. Lett. 93, 035901 (2004).
26. Lee, J. et al. Stabilization of graphene nanopore. Proc. Natl Acad. Sci. USA 111 7522-7526 (2014)

27. Hong, I.-P., Brun, C., Pivetta, M., Patthey, F. \& Schneider, W.-D. Coulomb blockade phenomena observed in supported metallic nanoislands. Front. Phys. 1, 13 (2013).

28. Romero, H. E. \& Drndic, M. Coulomb blockade and hopping conduction in PbSe quantum dots. Phys. Rev. Lett. 95, 156801 (2005).

29. Heiranian, M., Farimani, A. B. \& Aluru, N. R. Water desalination with a single-layer $\mathrm{MoS}_{2}$ nanopore. Nature Commun. 6, 8616 (2015).

\section{Acknowledgements}

This work was financially supported by the European Research Council (grant no. 259398, PorABEL), SNSF Consolidator grant (BIONIC BSCGI0_157802) and SNSF Sinergia Grant no. 147607. We thank the Centre Interdisciplinaire de Microscopie Électronique (CIME) at EPFL for access to electron microscopes and D. Alexander for help with Cs-corrected TEM (Titan Themis). We thank S. Marion and P. Leburton for their careful reading of our manuscript and for the helpful comments. Device fabrication was partially carried out at the EPFL Center for Micro/Nanotechnology (CMi).

\section{Author contributions}

J.F. conceived the idea, performed the measurements and interpreted the results. J.F. and K.L. fabricated the devices. J.F. and M.G. performed data analysis. D.D. and A.K. provided CVD-grown $\mathrm{MoS}_{2}$ samples. M.D.V. proposed the current-molarity relation. J.F. and A.R. designed the experiments and wrote the manuscript. A.R. supervised the work. All authors commented on the manuscript.

\section{Additional information}

Supplementary information is available in the online version of the paper. Reprints and permissions information is available online at www.nature.com/reprints.

Correspondence and requests for materials should be addressed to J.F. or A.R.

\section{Competing financial interests}

The authors declare no competing financial interests. 


\section{Methods}

Nanopore fabrication. The $\mathrm{MoS}_{2}$ nanopores are fabricated either using our recently reported atomic-scale nanopore fabrication technique based on controlled electrochemical reaction (ECR; ref. 13) or using electron irradiation ${ }^{12}$. Briefly, $\mathrm{SiN}_{x}$ membranes $(10 \mu \mathrm{m} \times 10 \mu \mathrm{m}$ to $50 \mu \mathrm{m} \times 50 \mu \mathrm{m}, 20 \mathrm{~nm}$ thick $)$ are manufactured using $\mathrm{KOH}$ wet etching. Focused ion beam (FIB) irradiation is applied to drill a 50-300-nm opening on that membrane. CVD-grown single-layer $\mathrm{MoS}_{2}$ (ref. 30) membranes are suspended on the FIB opening by transferring from sapphire substrates using a $\mathrm{MoS}_{2}$ transfer stage. ECR is done by applying a step-like transmembrane potential to the membrane and monitoring the transmembrane ionic current with a FEMTO DLPCA-200 amplifier (FEMTO Messtechnik GmbH). Nanopores are formed under the critical voltage $(>0.8 \mathrm{~V})$. The size estimation has an error bar of $0.1 \mathrm{~nm}$ with aberration-corrected TEM images and $0.2 \mathrm{~nm}$ with ECR pore formation ${ }^{13}$.

Ionic transport measurement. Ionic transport experiments are performed using the same set-up as described before ${ }^{12,13}$. After mounting in the polymethylmethacrylate (PMMA) chamber, the chips were wetted with
$\mathrm{H}_{2} \mathrm{O}$ :ethanol (1:1) for (30 min-24 h to rule out hydrophobic effects). Ionic transport is performed by taking the current-voltage response ( $I-V$ characteristic) of the nanopore in salty solutions (various ion types, ionic concentration or $\mathrm{pH}$ ) using an Axopatch 200B patch clamp amplifier (Molecular Devices) or a FEMTO DLPCA-200 amplifier for a higher bias than $1 \mathrm{~V}$. A pair of chlorinated Ag/AgCl electrodes is used to apply the voltage and detect the current. Current-voltage measurements ( $I-V$ characteristic) are performed by applying an alternating voltage sweep with a step size from 10 to $50 \mathrm{mV}$ and measuring the current (recording frequency 6,250 Hz) at same time. The current at each voltage value is measured for $10 \mathrm{~s}$ and the voltage range is typically set to -800 to $800 \mathrm{mV}$. The $I-V$ curve is then plotted using the voltage values and corresponding fitted steady-state current. The measured error bar is below $1 \%$ and therefore not plotted. Crosscheck experiments have been done to make sure the pore is not enlarged.

\section{References}

30. Dumcenco, D. et al. Large-area epitaxial monolayer $\mathrm{MoS}_{2}$. ACS Nano 9, 4611-4620 (2015) 